\title{
Temporal variation of wing geometry in Aedes albopictus
}

\author{
Paloma Oliveira Vidal ${ }^{1,3} /{ }^{+}$, Eneas Carvalho ${ }^{2}$, Lincoln Suesdek ${ }^{1,3}$ \\ ${ }^{1}$ Seção Parasitologia ${ }^{2}$ Centro de Biotecnologia, Instituto Butantan, São Paulo, SP, Brasil ${ }^{3}$ Programa de Pós-Graduação em \\ Biologia da Relação Patógeno-Hospedeiro, Instituto de Ciências Biomédicas, Universidade de São Paulo, São Paulo, SP, Brasil
}

\begin{abstract}
Although native to the tropical and subtropical areas of Southeast Asia, Aedes albopictus is now found on five continents, primarily due to its great capacity to adapt to different environments. This species is considered a secondary vector of dengue virus in several countries. Wing geometric morphometrics is widely used to furnish morphological markers for the characterisation and identification of species of medical importance and for the assessment of population dynamics. In this work, we investigated the metric differentiation of the wings of Ae. albopictus samples collected over a four-year period (2007-2010) in São Paulo, Brazil. Wing size significantly decreased during this period for both sexes and the wing shape also changed over time, with the wing shapes of males showing greater differences after 2008 and those of females differing more after 2009. Given that the wings play sex-specific roles, these findings suggest that the males and females could be affected by differential evolutionary pressures. Consistent with this hypothesis, a sexually dimorphic pattern was detected and quantified: the females were larger than the males (with respect to the mean) and had a distinct wing shape, regardless of allometric effects. In conclusion, wing alterations, particularly those involving shape, are a sensitive indicator of microevolutionary processes in this species.
\end{abstract}

Key words: geometric morphometrics - microevolution - Culicidae - mosquitoes - Aedes albopictus

Aedes (Stegomyia) albopictus (Skuse 1894) (Diptera: Culicidae), commonly known as the Asian tiger mosquito, originated in the forests of Southeast Asia and has now spread to America, Africa, the Middle East and Europe, largely due to human activities (Gratz 2004, WHO 2012). This species is an epidemiologically important vector for the transmission of many viral pathogens, such as dengue fever, yellow fever virus, West Nile virus and St. Louis encephalitis virus (Fernández et al. 2012). This mosquito was also recently confirmed to have been the primary vector for Chikungunya virus, a pathogen originally from Africa, during the 2007 outbreaks in Italy and Gabon (Paupy et al. 2009).

The spread of Ae. albopictus throughout the world began in the 1970s (Benedict et al. 2007) and this mosquito was first recorded in Brazil in the 1980s (Forattini 1986). This species is currently considered the most invasive mosquito in the world and control is difficult owing to the rapid reproduction of the species and its capacity to adapt to different environments.

The efficacy of the control procedures for Aedes spp depends on many contextual features, with microevolution representing one relevant limiting factor. For instance, the evolutionary changes within a species may produce populations that are resistant to insecticides (Braga et al. 2004, da-Cunha et al. 2005, Gómez et al. 2011, Fontoura et al. 2012) or may alter their vectorial

Financial support: CNPQ (134717/2009-0), FAPESP (2010/15039-1) +Corresponding author: lomavidal@gmail.com

Received 11 July 2012

Accepted 14 September 2012 capacity (Almeida et al. 2005, Freitas 2010). Although microevolution has been investigated in the congeneric dengue vector Aedes aegypti (Lourenço-de-Oliveira et al. 2004, Costa-Ribeiro et al. 2007, Vidal \& Suesdek 2012), the knowledge of microevolution in Ae. albopictus remains anecdotal.

Although the traditional approach to investigating the microevolution of culicids is based on genotypic markers (Lenormand et al. 1999, Lenormand \& Raymond 2000, Corley 2005), there is currently an increasing tendency to use phenotypic characters, primarily wing shape, to describe microevolutionary patterns (Dujardin 2008). The wings of culicids have been geometrically characterised using the low-cost "geometric morphometrics" method (Jirakanjanakit et al. 2008, Dujardin et al. 2009, Henry et al. 2010, Devicari et al. 2011, Vidal et al. 2011). Microevolutionary studies of the wings are feasible because of the strong heritability of the relevant phenotypic traits. Moreover, the wings are nearly bidimensional and can be used for geometrical and multivariate analyses.

Given that wing geometry is a powerful microevolutionary marker and that microevolutionary studies of $A e$. albopictus have not been conducted, our primary objective was to investigate the temporal variation in wing size and shape in samples of this species collected from the same location over a four-year period.

\section{MATERIALS AND METHODS}

Mosquitoes - Larvae of Ae. albopictus were collected from traps consisting of four open quarter-litre water containers (for a total volume of $1.000 \mathrm{~mL}$ ). A group of four containers (with 1 ovitrap per container) was placed in a one square metre patch in each of two locations in a park in São Paulo (SP), Brazil $\left(23.566^{\circ} \mathrm{S} 46.719^{\circ} \mathrm{W}\right)$, with a $200 \mathrm{~m}$ distance between each. Four chronological samples were collected during the rainy seasons from 
2007-2010 and were maintained in the laboratory under standard temperature and humidity conditions (25 \pm $\left.1{ }^{\circ} \mathrm{C} ; 80 \pm 10 \%\right)$. The emerging adults were identified at the species level (Forattini 2002) and were preserved in $70 \%$ ethanol. We analysed the left wings of 22 individuals collected in 2007, 53 individuals collected in 2008, 70 individuals collected in 2009 and 49 individuals collected in 2010.

Geometric data acquisition - The left wing of each individual was detached from the thorax and mounted with Canada balsam (Sigma, St. Louis, MO, USA) between a slide and a coverslip. Images of the wings were obtained using a Leica S6 stereoscopic microscope equipped with plain lenses, which diminish image distortion. The coordinates of 18 landmarks represented by vein intersections (Fig. 1) were obtained using TpsDig version 1.4 (Rohlf 2006).

Morphometric analyses - Chronological samples were compared with respect to wing size and shape to evaluate the morphological variation during the study period. Owing to the sexual dimorphism of the wings, the males and females were analysed separately.

To assess the overall wing sizes, the isometric estimator known as centroid size (Bookstein 1991) was computed from the landmark coordinates with the MOG V. 82 programme (Dujardin 2010). The scores of the centroid sizes were compared among samples with a parametric ANOVA and a post hoc Tukey test or T-test using Statistica 7.0 software (StatSoft, Tulsa, OK, USA).

The generalised least-squares Procrustes superimposition algorithm (Rohlf 1990) was used to produce shape variables (partial warps) and the canonical variations were used to examine the differences in the shape of the left wing of samples collected in the different years. The graphics were obtained using MorphoJ software (Klingenberg 2011) and the most influential landmarks were obtained with the COV programme (Dujardin 2010).

To explore the relationships among the Ae. albopictus samples collected over four years, pairwise Mahalanobis distances between the samples were calculated and compared using nonparametric permutation tests $(10,000$ randomisations) with MorphoJ software. To illustrate the morphological divergence among the samples, neighbour-joining phenograms were constructed using the PHYLIP - Phylogeny Inference Package version 3.6. (distributed by the author, J Felsenstein, 2005, Department of Genome Sciences, University of Washington, Seattle, WA).

Allometry was estimated with a multivariate regression of the Procrustes coordinates vs. the centroid size and the significance of the allometry was assessed by a permutation test with 10,000 randomisations using MorphoJ software. Reclassification tests were performed using the Mahalanobis distances as estimators of the metric distance. The distances were computed on discriminant axes that were estimated without the individual (wing) to be classified; the individual was only introduced afterwards (cross-validated classification, MorphoJ software (Klingenberg 2011). Voucher specimens were deposited in the entomological collection of the Butantan Institute, SP.

\section{RESULTS}

Size variation - The mean centroid sizes for the females (in mm) were 2.37 (in 2007), 2.67 (in 2008), 2.33 (in 2009) and 1.95 (in 2010) and those for the males were 2.33 (in 2007), 2.05 (in 2008), 1.96 (in 2009) and 1.78 (in 2010). Within each sex, all pairwise size comparisons indicated significant differences (ANOVA; $\mathrm{p}<0.05$ ), except for the transition between 2008-2009 for the males. The females were larger than the males in 2008 and 2009 ( $t$ test; $p<0.05$ ); the descriptive statistics for size are presented in Fig. 2. In terms of allometry, the contribution of size to the shape variation was statistically significant $(\mathrm{p}<0.001$; both sexes): the proportion of the variance in shape explained by size was $6 \%$ for females and $5 \%$ for males. Although weak, the allometry was removed from the shape analyses.

Shape variation - After the removal of the allometry, a canonical variate analysis revealed differences in wing shape over the years 2007-2010 (Fig. 3). The greatest divergence occurred between 2009-2010 (for both sexes), whereas the least divergence occurred between 20072008 (for males).

The comparison of the wing shape consensus after the Procrustes superimposition revealed a landmark dis-

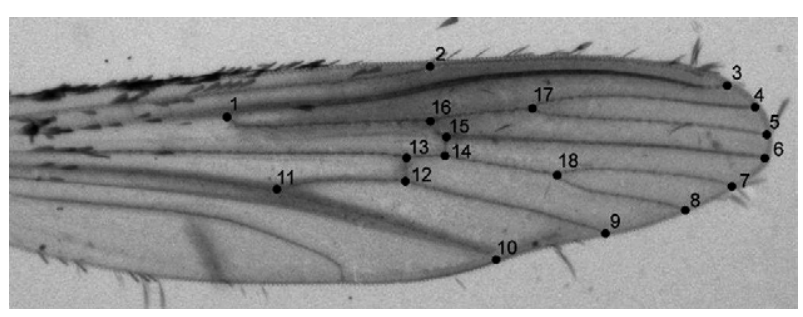

Fig. 1: wing of Aedes albopictus (female) showing the 18 landmarks chosen for morphometrics analysis.

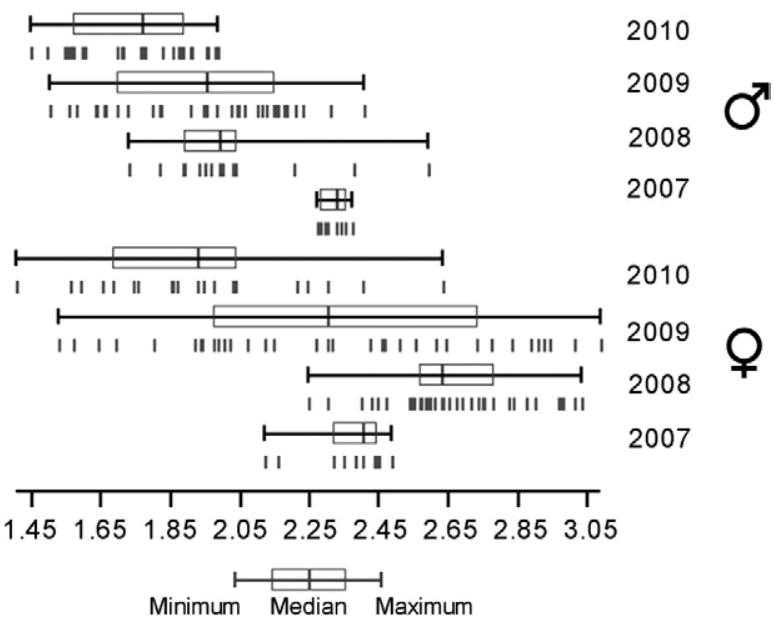

Fig. 2: descriptive statistics of wing centroid sizes (in mm) of males and females from different years. Small vertical bars indicate individuals. 
placement over the study period (Fig. 4). The most influential landmarks were \#10 for the females and \#2 for the males (Fig. 4).

The scores of the Mahalanobis distances between the yearly samples ranged from 3.04-5.33 (females) and from 3.42-5.00 (males). The phenograms of those pairwise distances (Fig. 5) corroborated the similarity patterns among the chronological groups observed in the canonical variate analysis (Fig. 3). Permutation tests revealed that the shape divergence was extremely significant ( $\mathrm{p}$ $<0.0001)$ in all comparisons. The cross-validated reclassification accuracy based on the Mahalanobis distances ranged from $50-100 \%$ among the females and from $66.6-$ $92.6 \%$ among the males (Table), with the scores increasing in a parallel fashion between the sexes during the four-year study period.

Wing shape sexual dimorphism was observed in all samples after the removal of the allometric effect of size, as shown by the morphospaces of canonical variables in
Fig. 6 (only years 2009 and 2010 are depicted). Similar results were obtained if the same analysis was repeated without the removal of allometry (Fig. 6).

\section{DISCUSSION}

To our knowledge, this is the first description of phenotypic variation in a natural population of Ae. albopictus over time. The results showed that wing morphology in Ae. albopictus may change over periods as short as one year and that this process is continuous. These results led us to believe that wing geometry is sensitive to microevolutionary processes, an interpretation that is in accordance with that predicted by Dujardin (2008) for culicids. Similar observations have also been reported for hemipteran insects (Dujardin et al. 2009).

Of the main primary components of wing form, it is probable that the shape is more directly related to microevolution in the present study. Given that shape in Aedes spp has been shown to be genetically determined and
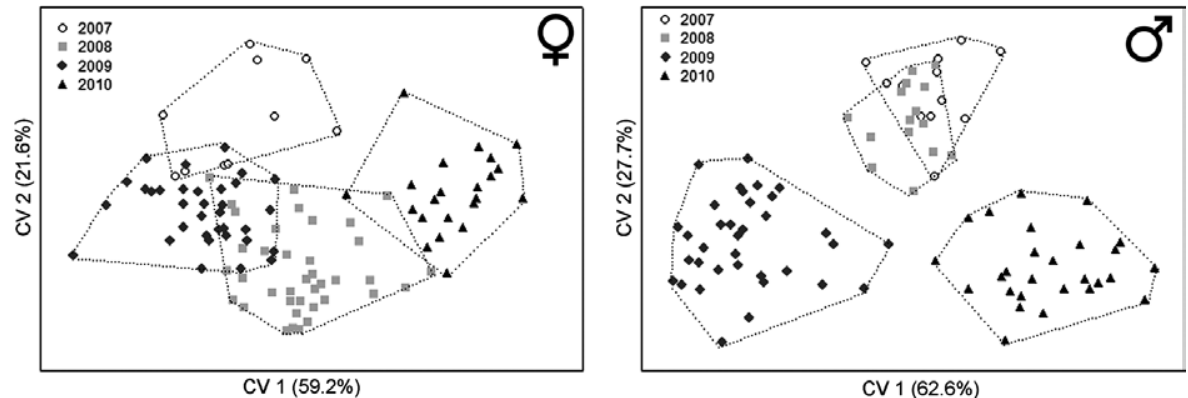

Fig. 3: morphological spaces of first two canonical variables (CV)1 and 2 originated from the comparison of wing shape across the years. Between brackets, the relative contribution of each $\mathrm{CV}$.
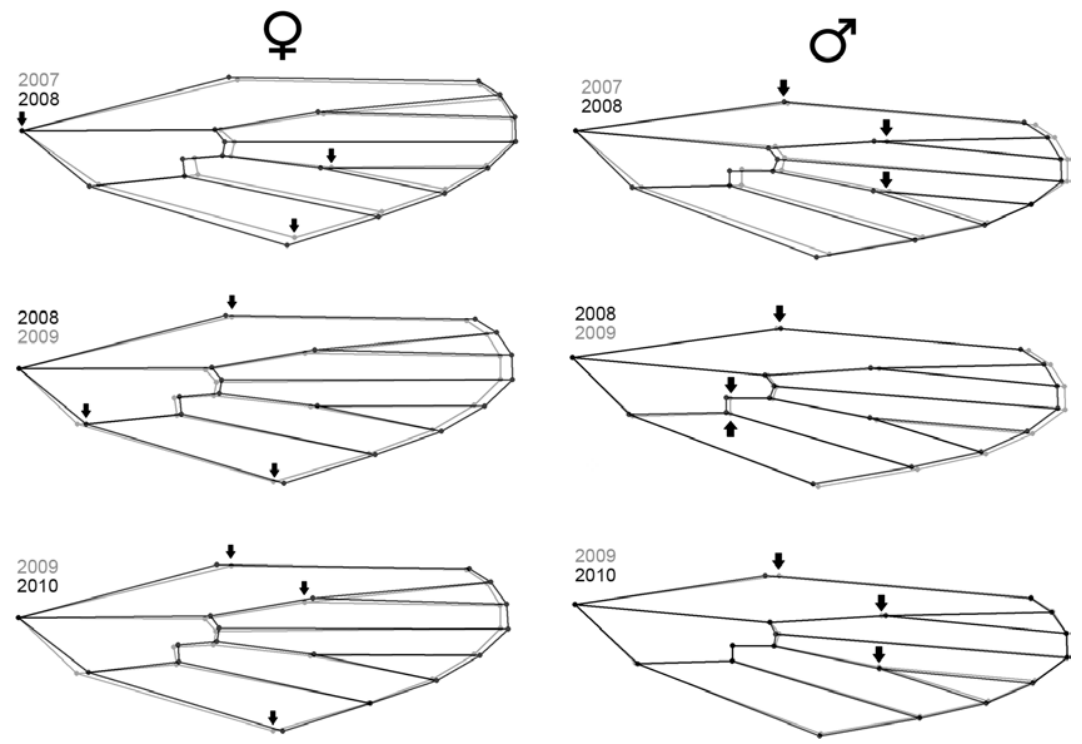

Fig. 4: wing shape consensus after Procrustes superimposition in each year aligned on landmark 1. Comparisons illustrate shape transition between 2007-2008, 2008-2009 and 2009-2010. Arrows indicate the landmarks of most influence on wing variation. 
heritable (Dujardin 2008, Henry et al. 2010, Klingenberg 2010), one could conclude that wing variation is concomitant with a general genotypic variation in Ae. albopictus.

It is remarkable that the shape divergence gradually increased over the four-year study period and that we observed a reclassification accuracy of up to $100 \%$. Arguably, both microevolution and population substitution may have occurred rapidly and it must now be investigated whether such genotypic fluctuations are primarily due to migration, drift or other processes. A deeper understanding of the microevolutionary meaning of our findings could improve surveillance programmes and aid in the development of genetically modified mosquitoes.

The male and female wings decreased in size over the entire four-year period. As concluded for the shape, the size of the wing may also be a manifestation of microevolutionary changes. However, certain environmental elements may strongly influence wing size, a phenomenon known as plasticity (West-Eberhard 1989, SchachterBroide et al. 2009). Because the samples were collected in the same location and during the same climatic season each year in this study, the population density and food availability are more likely to have influenced the size of the insects than the temperature and humidity. If part of the size variation was due to environmental changes, the shape variation could actually be partly due to plasticity. However, the low allometry and the elimination of its effects before performing the analyses allowed us to discard this hypothesis.

Unlike the wing shape, the wing size did not vary significantly between 2008-2009. These uncorrelated size and shape variation patterns appear to be due to the existence of distinct determinants for those biological variables, as discussed previously (Dujardin 2008, Klingenberg 2010).

Although we were not performing an a priori investigation of the differences between the sexes, we also observed disparities in wing shape, with different rates of change between the sexes, as previously discussed for wing size. For instance, the wing shape of the males changed less than that of the females between 20072008. The sexual dimorphism of the wing shape was also significant in all comparisons, even when the allometry was removed. Our interpretation of these results is that the evolution of wings is controlled by different factors in the two sexes, as already proposed by others for blood-feeding mosquitoes (Devicari et al. 2011, Vidal et al. 2011). It is plausible to consider that the wings of males and females do not follow the same evolutionary pathway in Ae. albopictus because these organs assume sex-specific roles in this species. Both sexes produce and recognise each other through courtship sounds (Duhrkopf \& Hartberg 1992) and the females must fly with precision to feed quickly on a host that is awake. These behaviours could result in sex-specific differences in selective pressures; thus, sexual selection cannot be discarded within this scenario.

In general, our results and interpretations support Dujardin's theory (2008) that wing geometry is a use-

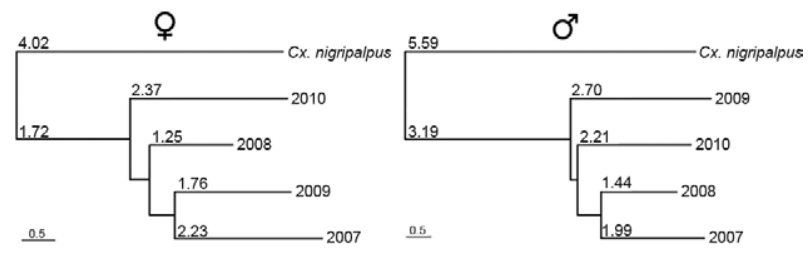

Fig. 5: neighbour-joining phenograms of Mahalanobis distances between each yearly sample, regarding wing shape. Culex nigripalpus was used as outgroup.

TABLE

Scores of reclassification tests after validation

\begin{tabular}{lccc}
\hline & & \multicolumn{2}{c}{ Cross-validation scores } \\
\cline { 3 - 4 } Comparison & Year & $\begin{array}{c}\text { Female } \\
(\%)\end{array}$ & $\begin{array}{c}\text { Male } \\
(\%)\end{array}$ \\
\hline 2007 vs. 2008 & 2007 & 50 & 66.6 \\
& 2008 & 73 & 73.3 \\
2008 vs. 2009 & 2008 & 81.6 & 80 \\
& 2009 & 77.1 & 77.1 \\
2009 vs. 2010 & 2009 & 91.4 & 80 \\
& 2010 & 100 & 92.6 \\
\hline
\end{tabular}

B
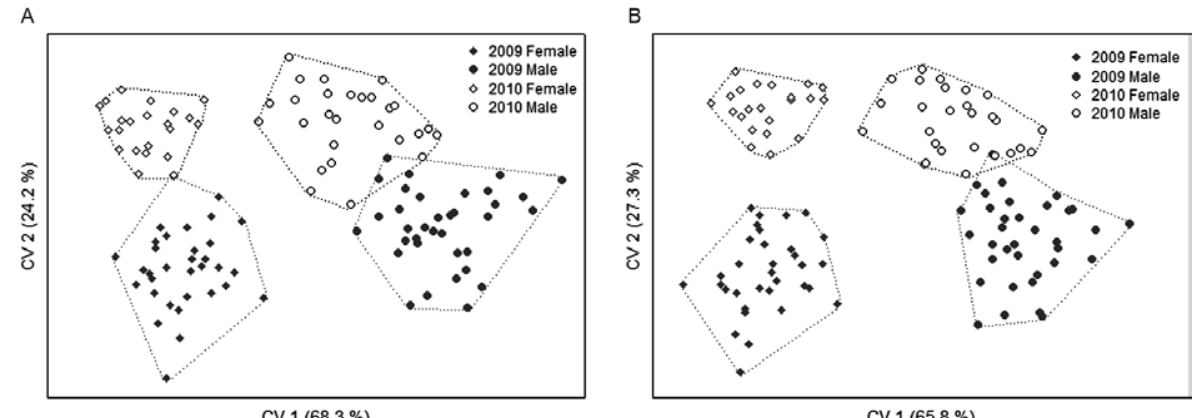

CV $1(65.8 \%)$

Fig. 6: morphological spaces of first two canonical variables (CV)1 and 2 originated from the comparison of wing shape between 2009-2010. A: without remotion of allometry; B: after remotion of allometric effect. Between brackets, the relative contribution of each CV. 
ful characteristic for detecting microevolutionary patterns and processes. Geometric morphometric analysis remains an effective and inexpensive tool to detect recent phenotypic and, arguably, genotypic changes. This tool is particularly useful for investigating Ae. albopictus, which is an invasive and plastic vector. As a result of this investigation, some new complex questions have arisen, confirming that this approach opens promising opportunities for further research.

\section{ACKNOWLEDGEMENTS}

To Dr Jean-Pierre Dujardin, whose comments helped improve the paper, and to Cristina Chagas, for technical support.

\section{REFERENCES}

Almeida AP, Baptista SS, Sousa CA, Novo MT, Ramos HC, Panella NA, Godsey M, Simões MJ, Anselmo ML, Komar N, Mitchell CJ, Ribeiro H 2005. Bioecology and vectorial capacity of Aedes albopictus (Diptera: Culicidae) in Macao, China, in relation to dengue virus transmission. J Med Entomol 42: 419-428.

Benedict M, Levine RS, Hawley WA, Lounibos LP 2007. Spread of the tiger: global risk of invasion by the mosquito Aedes albopictus. Vector Borne Zoonotic Dis 7: 76-85.

Bookstein FL 1991. Morphometric tools for landmark data: geometry and biology, Cambridge University Press, Cambridge, $435 \mathrm{pp}$.

Braga IA, Lima JBP, Soares S da S, Valle D 2004. Aedes aegypti resistance to temephos during 2001 in several municipalities in the states of Rio de Janeiro, Sergipe and Alagoas, Brazil. Mem Inst Oswaldo Cruz 99: 199-203.

Corley LS 2005. Microevolution and development: studies of the genetic basis of adaptive variation in insects. Evol Dev 7: 79-80.

Costa-Ribeiro MCV, Lourenço-de-Oliveira R, Failloux AB 2007. Low gene flow of Aedes aegypti between dengue-endemic and dengue-free areas in southeastern and southern Brazil. Am J Trop Med Hyg 77: 303-309.

da-Cunha MP, Lima JBP, Brogdon WG, Moya GE, Valle D 2005. Monitoring of resistance to the pyrethroid cypermethrin in Brazilian Aedes aegypti (Diptera: Culicidae) populations collected between 2001 and 2003. Mem Inst Oswaldo Cruz 100: 441-444.

Devicari M, Lopes AR, Suesdek R 2011. Dimorfismo sexual alar em Aedes scapularis (Diptera: Culicidae). Biota Neotrop 11: 165-169.

Duhrkopf RE, Hartberg WK 1992. Differences in male mating response and female flight sounds in Aedes aegypti and Ae. Albopictus (Diptera: Culicidae). J Med Entomol 29: 796-801.

Dujardin JP 2008. Morphometrics applied to medical entomology. Infect Genet Evol 8: 875-890.

Dujardin JP 2010. COO, MOG and COV for Windows, Institut de Recherches pour le Développement, Marseille.

Dujardin JP, Costa J, Bustamante D, Jaramillo N, Catalá S 2009. Deciphering morphology in Triatominae: the evolutionary signals. Acta Trop 110: 101-111.

Fernández MCM, Jean YS, Callaba CAF, López LS 2012. The first report of Aedes (Stegomyia) albopictus in Haiti. Mem Inst Oswaldo Cruz 107: 279-281.

Fontoura NG, Bellinato DF, Valle D, Lima JBP 2012. The efficacy of a chitin synthesis inhibitor against field populations of organophosphate-resistant Aedes aegypti in Brazil. Mem Inst Oswaldo Cruz 107: 387-395.
Forattini OP 1986. Aedes (Stegomyia) albopictus (Skuse) identification in Brazil. Rev Saude Publica 20: 244-245.

Forattini OP 2002. Culicidologia médica, EDUSP, São Paulo, 864 pp.

Freitas RM 2010. A review on the ecological determinants of Aedes aegypti (Diptera: Culicidae) vectorial capacity. Oecologia 14: 726-736.

Gómez A, Seccacini E, Zerba E, Licastro S 2011. Comparison of the insecticide susceptibilities of laboratory strains of Aedes aegypti and Aedes albopictus. Mem Inst Oswaldo Cruz 106: 993-996.

Gratz NG 2004. Critical review of the vector status of Aedes albopictus. Med Vet Entomol 18: 215-227.

Henry A, Thongsripong P, Fonseca-Gonzalez I, Jaramillo-Ocampo N, Dujardin JP 2010. Wing shape of dengue vectors from around the world. Infect Genet Evol 10: 207-214.

Jirakanjanakit N, Leemingsawat S, Dujardin JP 2008. The geometry of the wing of Aedes (Stegomyia) aegypti in isofemale lines through successive generations. Infect Gen Evol 8: 414-421.

Klingenberg CP 2010. Evolution and development of shape: integrating quantitative approaches. Nat Rev Genet 11: 623-635.

Klingenberg CP 2011. MORPHOJ: an integrated software package for geometric morphometrics. Mol Ecol Resour 11: 353-357.

Lenormand T, Bourguet D, Guillemaud T, Raymond M 1999. Tracking the evolution of insecticide resistance in the mosquito Culex pipiens. Nature 400: 861-864.

Lenormand T, Raymond M 2000. Analysis of clines with variable selection and variable migration. Am Nat 155: 70-82.

Lourenço-de-Oliveira R, Vazeille M, Filippis AMB, Failloux AB 2004. Aedes aegypti in Brazil: genetically differentiated populations with high susceptibility to dengue and yellow fever viruses. Trans R Soc Trop Med Hyg 98: 43-54.

Paupy C, Delatte H, Bagny L, Corbel V, Fontenille D 2009. Aedes albopictus, an arbovirus vector: from the darkness to the light. Microbes Infect 11: 1177-1185.

Rohlf FJ 1990. Rotational fit (Procrustes) methods. In FJ Rohlf, FL Bookstein (eds.), Proceedings of the Michigan morphometrics workshop, University of Michigan Museum of Zoology, Ann Arbor, 227-236.

Rohlf FJ 2006. TpsDig2, Digitize Landmarks and Outlines, version 2.10, Department of Ecololy \& Evolution, Stony Brook University, New York.

Schachter-Broide J, Gurtler RE, Kitron U, Dujardin JP 2009. Temporal variations of wing size and shape of Triatoma infestans (Hemiptera: Reduviidae) populations from northwestern Argentina using geometric morphometry. J Med Entomol 46: 994-1000.

Skuse FAA 1894. The banded mosquito of Bengal. Indian Museum Notes 3: 20.

Vidal PO, Peruzin MC, Suesdek L 2011. Wing diagnostic characters for Culex quinquefasciatus and Culex nigripalpus (Diptera: Culicidae). Rev Bras Entomol 55: 134-137.

Vidal PO, Suesdek L 2012. Comparison of wing geometry data and genetic data for assessing the population structure of Aedes aegypti. Infect Genet Evol 12: 591-596.

West-Eberhard M 1989. Phenotypic plasticity and the origins of diversity. Annu Rev Ecol Evol Syst 20: 249-278.

WHO - World Health Organization 2012. Dengue and severe dengue. Available from: who.int/mediacentre/factsheets/fs117/en/. 